Article

\title{
Spontaneous Emission Enhancement by a Rectangular-Aperture Optical Nanoantenna: An Intuitive Semi-Analytical Model of Surface Plasmon Polaritons
}

\author{
Xinyue Zhang ${ }^{1}$, Xuelin Zhai ${ }^{1}$, Can Tao ${ }^{1}$, Ning Wang ${ }^{1}$, Ying Zhong ${ }^{2}$ and Haitao Liu ${ }^{1, *(\mathbb{D})}$ \\ 1 Tianjin Key Laboratory of Micro-Scale Optical Information Science and Technology, Institute of Modern \\ Optics, College of Electronic Information and Optical Engineering, Nankai University, Tianjin 300350, China; \\ zhangxinyue_thera@163.com (X.Z.); zx1310326@126.com (X.Z.); taocancan@sina.cn (C.T.); \\ wangning@mail.nankai.edu.cn (N.W.) \\ 2 State Key Laboratory of Precision Measurement Technology and Instruments, Tianjin University, \\ Tianjin 300072, China; yzhong@tju.edu.cn \\ * Correspondence: liuht@nankai.edu.cn
}

check for updates

Citation: Zhang, X.; Zhai, X.; Tao, C.; Wang, N.; Zhong, Y.; Liu, H.

Spontaneous Emission Enhancement by a Rectangular-Aperture Optical Nanoantenna: An Intuitive

Semi-Analytical Model of Surface

Plasmon Polaritons. Photonics 2021, 8 , 572. https://doi.org/10.3390/ photonics 8120572

Received: 22 November 2021 Accepted: 10 December 2021 Published: 12 December 2021

Publisher's Note: MDPI stays neutral with regard to jurisdictional claims in published maps and institutional affiliations.

Copyright: (c) 2021 by the authors. Licensee MDPI, Basel, Switzerland. This article is an open access article distributed under the terms and conditions of the Creative Commons Attribution (CC BY) license (https:// creativecommons.org/licenses/by/ $4.0 /)$.

\begin{abstract}
The spontaneous-emission enhancement effect of a single metallic rectangular-aperture optical nanoantenna on $\mathrm{SiO}_{2}$ substrate was investigated theoretically. By considering the excitation and multiple scattering of surface plasmon polaritons (SPPs) in the aperture, an intuitive and comprehensive SPP model was established. The model can comprehensively predict the total spontaneous emission rate, the radiative emission rate and the angular distribution of the far-field emission of a point source in the aperture. Two phase-matching conditions are derived from the model for predicting the resonance and show that the spontaneous-emission enhancement by the antenna comes from the Fabry-Perot resonance of the SPP in the aperture. In addition, when scanning the position of the point source and the aperture length, the SPP model does not need to repeatedly solve the Maxwell's equations, which shows a superior computational efficiency compared to the full-wave numerical method.
\end{abstract}

Keywords: optical nanoantenna; spontaneous emission enhancement; surface plasmon polariton; semi-analytical model

\section{Introduction}

In the field of life sciences, particle-type optical nanoantennas [1-4] and aperturetype optical nanoantennas [5-10] can achieve single-molecule fluorescence detection even under high concentrations in physiological environments [9-11]. Optical nanoantennas support surface plasmons polaritons (SPPs) with a mode volume well below the diffraction limit $[12,13]$; therefore, they enable an enhancement of the electromagnetic field and spontaneous emission rate, which can increase the fluorescence intensity $[2,6,14]$. Compared with particle-type nanoantennas, aperture-type nanoantennas can obtain an excitation region that breaks the diffraction limit during the fluorescence excitation and can shield the background fluorescence outside the aperture during the fluorescence collection. Therefore, aperture-type nanoantennas are more conducive to the detection of single-molecule fluorescence under high concentrations [6-9].

According to whether the antenna supports resonance, the aperture-type nanoantennas can be divided into non-resonant aperture nanoantennas [8,10,15-18] and resonant aperture nanoantennas [19-24]. For example, the zero-mode waveguide $[5,8,10,15,16]$ is a non-resonant aperture nanoantenna. Compared with non-resonant aperture nanoantennas, resonant aperture nanoantennas can achieve a stronger fluorescence-excitation electric field and a higher spontaneous emission rate [6,19-23,25-31]. Until now, a variety of aperture nanoantennas that can support resonance have been proposed, such as circular-aperture nanoantennas [27,28], bowtie-aperture nanoantennas [21,22], an antenna in a box [29-31], 
rectangular-aperture nanoantennas $[6,19,20,23,25,26]$, etc. Among them, a rectangularaperture nanoantenna is one of the most basic structures. Thus, it can be of great theoretical significance to investigate the mechanism of the resonance and its resultant spontaneous emission enhancement for the rectangular-aperture nanoantenna.

In previous theoretical works of resonant aperture nanoantennas, the resonance enhancement properties of the antenna are attributed to the excitation of localized surface plasmon resonance (LSPR) [6,19-21,24,28,29], for which resonance will occur when the excitation frequency matches the resonance frequency of the LSPR. The LSPR can be defined as the quasinormal mode (QNM), which is the eigensolution of source-free Maxwell's equations and corresponds to a complex resonance/eigen frequency [32,33]. Moreover, the resonant aperture nanoantenna can be treated as an equivalent circuit composed of resistance, inductance and capacitance, and the antenna resonance can be predicted by considering the resonance of the alternating current in the circuit [34-36]. However, in the above models, the SPP does not appear in an explicit form. Some works assume that the SPP in the aperture nanoantenna forms a Fabry-Perot resonance, and then provide the conditions of antenna resonance $[23,26]$. In these models, some parameters (such as the SPP propagation constant and reflection coefficient) are obtained by fitting experimental data [23] or numerical simulation results [26].

In this paper, we investigate the fundamental resonant rectangular-aperture nanoantenna, and establish an SPP model by considering the excitation and multiple scattering of the SPP in the aperture in order to clarify the role of the SPP in the spontaneous emission enhancement by the antenna. All the parameters in the model are calculated based on the first principle of Maxwell's equations, which ensures that the model has a rigorous electromagnetic foundation and can give quantitative predictions. The model can comprehensively predict the total spontaneous emission rate, the radiative emission rate and the angular distribution of the far-field emission of a point source in the aperture. Two phase-matching conditions are derived from the model for predicting the resonance and show that the enhancement of spontaneous emission by the antenna comes from a FabryPerot resonance of the SPP in the aperture. Moreover, the SPP model also shows superior computational efficiency compared with the full-wave numerical method.

This article is organized as follows. In Section 2, the SPP model is introduced. In Section 3, the predictions of the model in comparison to the full-wave numerical results are discussed. Conclusions are summarized in Section 4.

\section{Methods}

The considered aperture antenna is composed of a single rectangular nanoaperture in gold film on a $\mathrm{SiO}_{2}$ substrate, and the medium inside and above the aperture is water, as shown in Figure 1a. The aperture has a length $L$ and a rectangular cross section with a width $D$ and a depth $h$ (i.e., the gold film thickness, which is set to be $50 \mathrm{~nm}$ unless otherwise specified).

A point electric-current source is set in the aperture and represents a fluorescent molecule $[2,14]$. The point source is set to be polarized in the $y$ direction, which can achieve the highest spontaneous emission rate compared with other polarization directions. The refractive indices of the $\mathrm{SiO}_{2}$ substrate and water are $n_{\mathrm{s}}=1.5$ and $n_{\mathrm{W}}=1.333$, respectively. The wavelength-dependent refractive index $n_{\mathrm{m}}$ of gold is given by a Drude model, $n_{\mathrm{m}}(\lambda)^{2}=\varepsilon_{\infty}-\lambda_{p}^{-2} /\left[\lambda^{-1}\left(\lambda^{-1}+i \lambda_{\gamma}^{-1}\right)\right]$, where $\varepsilon_{\infty}=8.842, \lambda_{p}=0.164 \mu \mathrm{m}$ and $\lambda_{\gamma}=20.689 \mu \mathrm{m}$ are determined by fitting the tabulated data (from visible to far-infrared wavelengths) in Ref. [37] $\left(n_{\mathrm{m}}=0.169+5.331 i\right.$ at wavelength $\lambda=1 \mu \mathrm{m}$, which is adopted throughout the paper). 
(a)

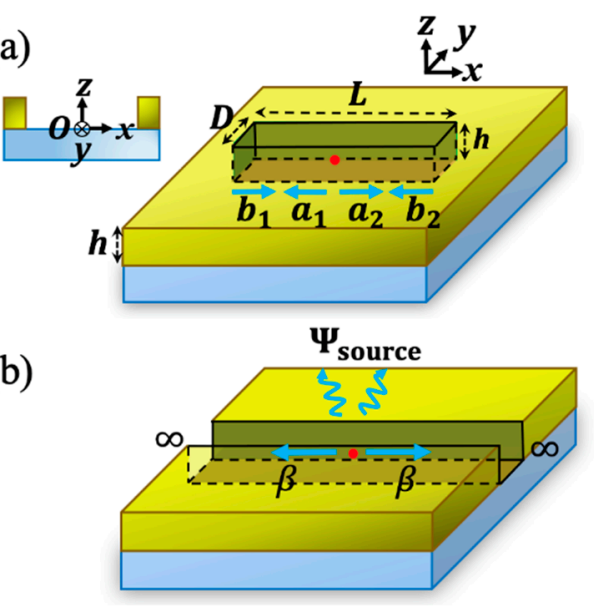

(c)

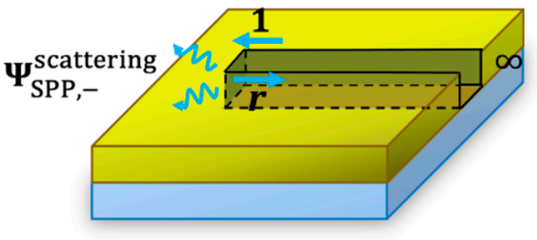

(d)

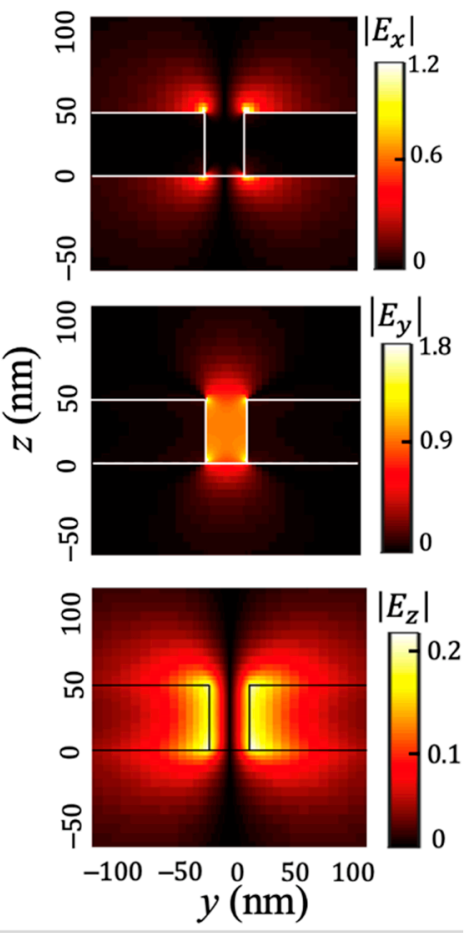

Figure 1. (a) Schematic diagram of the rectangular-aperture nanoantenna and the unknown SPP coefficients $a_{1}, a_{2}, b_{1}$ and $b_{2}$ in the SPP model. (b,c) Definitions of coefficients $\beta, r$ and electromagnetic fields $\boldsymbol{\Psi}_{\text {source }}$ and $\mathbf{\Psi}_{\mathrm{SPP},-}^{\text {scattering }}$ used in the SPP model. (d) Distribution of the electric-field components $\left(\left|E_{x}\right|,\left|E_{y}\right|,\left|E_{z}\right|\right)$ of the fundamental SPP mode on the transversal $y-z$ plane. The $y$ component of the SPP electric field $E_{y}$ is normalized to be 1 at $(y, z)=(0, h / 2)$. The superimposed solid lines represent the boundaries of the aperture. The results are calculated with the full-wave a-FMM for $D=30 \mathrm{~nm}$ and $h=50 \mathrm{~nm}$ at wavelength $\lambda=1 \mu \mathrm{m}$.

The $y$-polarized electric-current point source can be expressed as a current density $\mathbf{J}=\mathbf{y} \delta\left(x-x_{s}, y-y_{s}, z-z_{s}\right)$, where $\left(x_{s}, y_{s}, z_{s}\right)=(-L / 2+d, 0, h / 2)$ is the point-source coordinates, $d$ is the distance from the point source to the left termination of the aperture, $\delta$ is the Dirac function and $\mathbf{y}$ is the unit vector in the $y$ direction. The origin $O$ of the coordinate system is set at the center of the aperture on the surface of the $\mathrm{SiO}_{2}$ substrate. The total spontaneous emission rate of the point source can be expressed as $\Gamma_{\text {total }}=-\operatorname{Re}\left[E_{y}\left(x_{s}, y_{s}, z_{s}\right)\right] / 2$ [38], where $\operatorname{Re}\left[E_{y}\left(x_{s}, y_{s}, z_{s}\right)\right]$ denotes the real part of the $y$ component of the electric field at the pointsource position. The total emission rate $\Gamma_{\text {total }}$ contains the following two parts: the far-field radiative emission rate $\Gamma_{\mathrm{rad}}$ and the non-radiative rate $\Gamma_{\mathrm{nr}}$, i.e., $\Gamma_{\text {total }}=\Gamma_{\mathrm{rad}}+\Gamma_{\mathrm{nr}}$, where $\Gamma_{\text {rad }}$ is radiated to the far-field free space in the form of photons and $\Gamma_{\mathrm{nr}}$ is lost in the metal in the form of heat. The $\Gamma_{\mathrm{rad}}$ can be calculated as $\Gamma_{\mathrm{rad}}=\iint_{A} \mathbf{S} \cdot \mathbf{n} d a$, where $A$ is an arbitrary closed surface encircling the antenna and the point source, $\mathbf{n}$ is the outward-pointing unitary vector on $A$ and $\mathbf{S}$ is the time-averaged Poynting vector of the electromagnetic field excited by the point source.

To characterize the enhancement of the spontaneous emission rate, the enhancement factors of total and radiative emission rates are defined as $\gamma_{\text {total }}=\Gamma_{\text {total }} / \Gamma_{\text {water }}$ and $\gamma_{\mathrm{rad}}=\Gamma_{\mathrm{rad}} / \Gamma_{\mathrm{water}}$, respectively. Here, $\Gamma_{\mathrm{water}}=\eta_{\mathrm{vac}} n_{\mathrm{w}} k_{0}^{2} /(12 \pi)$ is the spontaneous emission rate of the point source in a homogeneous reference material (which is water for the present case, or other materials with a refractive index of $n_{\mathrm{w}}$ for other applications), with $\eta_{\mathrm{vac}}$ being the wave impedance in a vacuum and $k_{0}=2 \pi / \lambda$ being the wavenumber. $\gamma_{\text {total }}$ is also called the Purcell factor [14,39]. For high-speed nanoscale light sources (such as singlephoton sources) [40,41], the fluorescence lifetime can be shortened by achieving a high value of $\gamma_{\text {total }}$. For applications such as surface enhanced fluorescence or Raman-scattering molecular sensing [14,42-44] and super-bright light sources [40,41,45,46], the fluorescence intensity can be enhanced by improving the value of the quantum yield $\eta=\Gamma_{\text {rad }} /\left(\Gamma_{\text {total }}\right.$ 
$\left.+\Gamma_{\text {abs }}\right)=\gamma_{\text {rad }} /\left(\gamma_{\text {total }}+\eta_{0}^{-1}-1\right)[2,44]$, where $\eta_{0}=\Gamma_{\text {water }} /\left(\Gamma_{\text {water }}+\Gamma_{\text {abs }}\right) \in(0,1)$ denotes the intrinsic quantum yield of fluorescent emitters $\left(\Gamma_{\text {abs }}\right.$ represents the intrinsic loss rate of the emitters in the homogeneous reference material). When $\eta_{0} \approx 1$ (such as quantum dots [14]), there is $\eta \approx \gamma_{\text {rad }} / \gamma_{\text {total }}$ (called the radiation efficiency of the antenna [2]), and when $\eta_{0}$ is very low, there is $\eta \approx \eta_{0} \gamma_{\text {rad }}$.

To obtain rigorous data of spontaneous emission enhancement, a full-wave aperiodicFourier modal method (a-FMM) was adopted [47,48]. Then, to analyze the physical mechanism of spontaneous emission enhancement of the antenna, a semi-analytical SPP model was established based on an intuitive excitation and multiple scattering process of SPPs. In this model, the rectangular-aperture nanoantenna was treated as a waveguide along the $x$ direction. Since the size of the cross section of the nanoaperture is much smaller than the wavelength, only the fundamental SPP mode is propagative (with an almost real propagation constant) and bounded (field decaying to null in the transversal $y$ and $z$ directions) and thus is considered in the model, while all other higher-order waveguide modes are either evanescent or unbounded [49] and are neglected in the model. The field distribution of the fundamental SPP mode on the $y-z$ cross section was calculated by the full-wave a-FMM [Figure 1d], indicating that the dominant electric-field component $E_{y}$ was concentrated within the rectangular aperture.

As shown in Figure 1a, suppose the $y$-polarized point source is located at the position of the red dot in the aperture. We use $a_{1}, a_{2}, b_{1}, b_{2}$ to denote the unknown coefficients of the fundamental SPP modes propagating in the $x$ direction in the aperture. Considering the excitation and multiple scattering of the SPPs, the following set of SPP-coupling equations can be written:

$$
\begin{gathered}
a_{1}=\beta+b_{2} u_{2}, \\
b_{1}=a_{1} u_{1} r, \\
a_{2}=\beta+b_{1} u_{1}, \\
b_{2}=a_{2} u_{2} r .
\end{gathered}
$$

In Equations (1)-(4), $u_{1}=\exp \left(i k_{0} n_{\mathrm{eff}} d\right)$ and $u_{2}=\exp \left[i k_{0} n_{\mathrm{eff}}(L-d)\right]$ are the phase-shift factors of the SPP accumulated when SPP propagates from the point source to the left and right ends of the aperture, respectively; $n_{\mathrm{eff}}$ is the complex effective index of the SPP mode; and $r$ is the reflection coefficient of the SPP at one end of the aperture. $r$ and $n_{\text {eff }}$ can be calculated with the full-wave a-FMM $[47,48] . \beta$ is the coefficient of the SPP excited by the point source, and can be calculated using the following reciprocity theorem [50]:

$$
\beta=\frac{-\mathbf{p} \cdot \mathbf{E}_{\mathrm{SPP}}^{-}\left(0, y_{s}, z_{s}\right)}{\int_{-\infty}^{\infty} \int_{-\infty}^{\infty}\left[\mathbf{E}_{\mathrm{SPP}}^{-}\left(x_{0}, y, z\right) \times \mathbf{H}_{\mathrm{SPP}}^{+}\left(x_{0}, y, z\right)-\mathbf{E}_{\mathrm{SPP}}^{+}\left(x_{0}, y, z\right) \times \mathbf{H}_{\mathrm{SPP}}^{-}\left(x_{0}, y, z\right)\right] \cdot \mathbf{x} d y d z} .
$$

In Equation (5), $\mathbf{p}=\mathbf{y}$ is the unit vector in the polarization direction of the point source, $x_{0}$ can be selected arbitrarily and

$$
\Psi_{\mathrm{SPP}}^{ \pm}(x, y, z)=\Psi_{\mathrm{SPP}}^{ \pm}(0, y, z) \exp \left( \pm i k_{0} n_{\mathrm{eff}} x\right)
$$

denotes the electromagnetic field of the right-going $(+)$ and left-going $(-)$ fundamental SPP mode in the aperture, with $\Psi=[\mathbf{E}, \mathbf{H}]$ denoting both the electric $(\mathbf{E})$ and magnetic $(\mathbf{H})$ vectors. Equations (1)-(4) can be understood intuitively. For Equation (1), the left-going SPP with coefficient $a_{1}$ on the left side of the point source arises from the following two contributions: the first contribution from the direct excitation of the source (with excitation coefficient $\beta$ ), and the second contribution from the left-going SPP (with coefficient $b_{2}$ ) that propagates from the right end of the aperture to the left side of the point source (with a phase-shift factor $u_{2}$ ). For Equation (2), the right-going SPP (with coefficient $b_{1}$ ) on the left side of the point source originates from the reflection (reflection coefficient $r$ ) of the left-going SPP (with coefficient $a_{1}$ ) that propagates from the left side of the point source to the left end of the aperture (with a phase-shift factor $u_{1}$ ). Equations (3) and (4) can be 
understood similarly. Solving Equations (1)-(4), one can obtain the following analytical expressions of the SPP mode coefficients:

$$
\begin{gathered}
a_{1}=\frac{\beta\left(1+u_{2}^{2} r\right)}{1-u^{2} r^{2}}, \\
b_{1}=\frac{\beta\left(1+u_{2}^{2} r\right)}{1-u^{2} r^{2}} u_{1} r \\
a_{2}=\frac{\beta\left(1+u_{1}^{2} r\right)}{1-u^{2} r^{2}}, \\
b_{2}=\frac{\beta\left(1+u_{1}^{2} r\right)}{1-u^{2} r^{2}} u_{2} r,
\end{gathered}
$$

where $u=u_{1} u_{2}=\exp \left(i k_{0} n_{\text {eff }} L\right)$. Then, the electromagnetic field in the aperture can be expressed as follows:

$$
\mathbf{\Psi}_{\text {in }}(\mathbf{r})=\mathbf{\Psi}_{\text {source }}(\mathbf{r})+b_{1} \mathbf{\Psi}_{\mathrm{SPP}}^{+}(x+L / 2, y, z)+b_{2} \mathbf{\Psi}_{\mathrm{SPP}}^{-}(x-L / 2, y, z),
$$

where $\boldsymbol{\Psi}_{\text {source }}$ denotes the electromagnetic field excited by a point source in an infinitely long slot [Figure 1b] and can be calculated with the full-wave a-FMM [47,48]. The last two terms in Equation (11) represent the contributions of right-going and left-going SPPs, respectively. With Equation (11), the total emission rate of the point source can be obtained as follows:

$$
\Gamma_{\text {total }}=-\operatorname{Re}\left[E_{y, \text { source }}\left(x_{s}, y_{s}, z_{s}\right)+b_{1} u_{1} E_{y, \mathrm{SPP}}^{+}\left(0, y_{s}, z_{s}\right)+b_{2} u_{2} E_{y, \mathrm{SPP}}^{-}\left(0, y_{s}, z_{s}\right)\right] / 2 .
$$

To calculate the radiative emission rate with the SPP model, the electromagnetic field in the free space outside the antenna can be expressed as follows:

$$
\Psi_{\text {out }}(\mathbf{r})=\Psi_{\text {source }}(\mathbf{r})+a_{1} u_{1} \Psi_{\mathrm{SPP},-}^{\text {scattering }}(\mathbf{r})+a_{2} u_{2} \Psi_{\mathrm{SPP},+}^{\text {scattering }}(\mathbf{r}) \text {. }
$$

In Equation (13), $\Psi_{\mathrm{SPP},-}^{\text {scattering }}$ and $\Psi_{\mathrm{SPP},+}^{\text {scattering }}$ denote the scattered fields for incident unitary-coefficient left-going and right-going SPPs at the left and right ends of the aperture, respectively, and can be calculated with the full wave a-FMM. In the calculation, the scattered field is equal to the total field excited by the incident SPP mode minus the incident SPP field in an infinitely long slot. The first term of Equation (13) represents the field directly excited by the point source, and the second and third terms represent the scattered fields at both ends of the aperture from the two SPPs (with coefficients $a_{1}$ and $a_{2}$ and phase-shift factors $u_{1}$ and $u_{2}$ ) propagating away from the point source. Then, $\Gamma_{\text {rad }}$ can be calculated by Equation (13).

\section{Results and Discussion}

To analyze the spontaneous-emission enhancement properties of the antenna, we calculated the enhancement factors $\gamma_{\text {total }}=\Gamma_{\text {total }} / \Gamma_{\text {water }}$ and $\gamma_{\text {rad }}=\Gamma_{\text {rad }} / \Gamma_{\text {water }}$ of the total and radiative emission rates for different antenna lengths $L$. The point source is located at a distance $d=63 \mathrm{~nm}$ from the left end of the aperture or at the center of the aperture $(d=L / 2)$ with an excitation wavelength $\lambda=1 \mu \mathrm{m}$.

The full-wave a-FMM results in Figure 2 show that with the increase in $L$, the normalized total emission rate $\gamma_{\text {total }}$ (blue solid curves) and the normalized radiative emission rate $\gamma_{\text {rad }}$ (red solid curves) exhibit quasiperiodic resonance peaks with the peak values decreasing gradually. At the resonance peak positions, $\gamma_{\text {total }}$ and $\gamma_{\text {rad }}$ are far larger than one, indicating that the antenna can greatly enhance the total and radiative emission rates of the point source. When the gold-film thickness $h$ decreases from $50 \mathrm{~nm}$ (Figure 2a) to $30 \mathrm{~nm}$ (Figure $2 \mathrm{c}$ ), $\Gamma_{\text {total }}, \Gamma_{\text {rad }}$ and $\eta=\Gamma_{\text {rad }} / \Gamma_{\text {total }}$ (i.e., the radiation efficiency defined previously) do not change significantly, but the antenna lengths $L$ at the resonance peaks decrease. 
When the aperture width $D$ decreases from $30 \mathrm{~nm}$ (Figure $2 \mathrm{~b}$ ) to $10 \mathrm{~nm}$ (Figure $2 \mathrm{~d}$ ), $\Gamma_{\text {total }}$ and $\Gamma_{\text {rad }}$ increase, but their ratio $\eta=\Gamma_{\text {rad }} / \Gamma_{\text {total }}$ decreases. For an aperture width $D=30 \mathrm{~nm}$ and length $L=0.124 \mu \mathrm{m}$, the radiation efficiency $\eta$ can be up to $69.89 \%$ (with $\gamma_{\text {toal }}=658.81$ and $\left.\gamma_{\mathrm{rad}}=460.43\right)$. For $D=10 \mathrm{~nm}$ and $L=0.084 \mu \mathrm{m}, \gamma_{\text {toal }}$ and $\gamma_{\text {rad }}$ can reach 5152 and 1971, respectively (with $\eta=38.26 \%$ ).

(a)

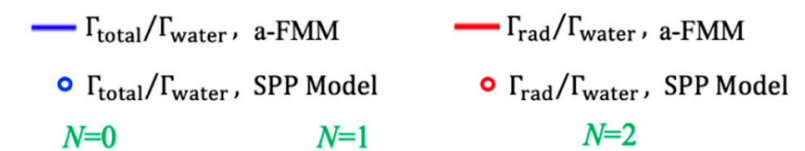

(b)

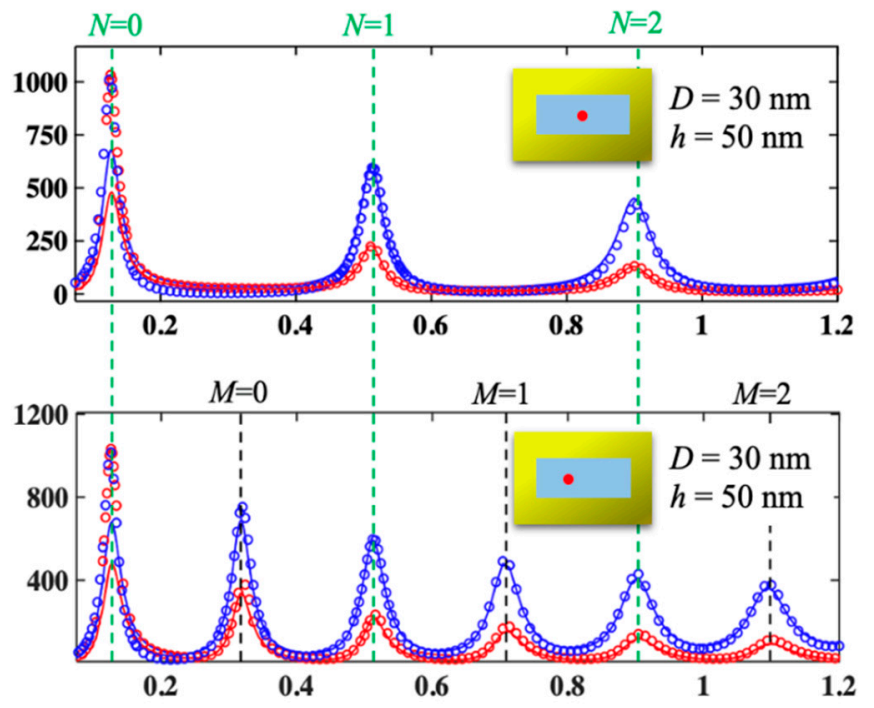

(c)

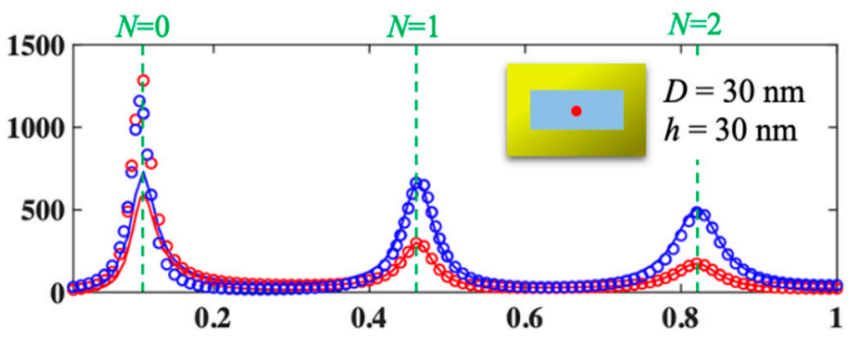

(d)

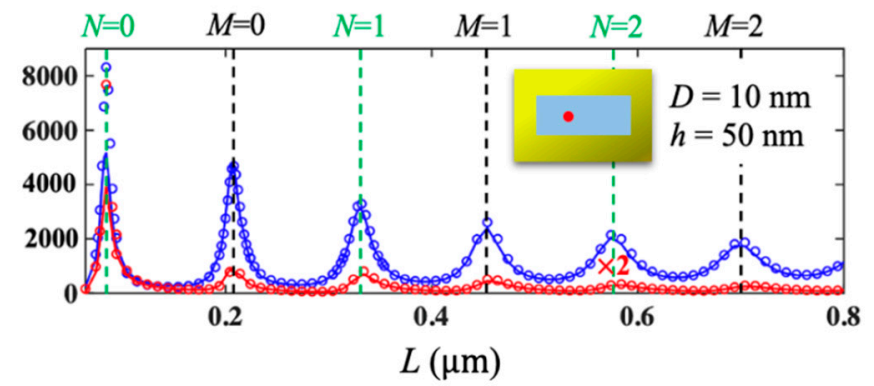

Figure 2. Normalized total and radiative emission rates (i.e., enhancement factors) $\Gamma_{\text {total }} / \Gamma_{\text {water }}$ (blue curves) and $\Gamma_{\text {rad }} / \Gamma_{\text {water }}$ (red curves) plotted as functions of the antenna length $L$ at an excitation wavelength $\lambda=1 \mu \mathrm{m}$. The solid curves and circles show the full-wave a-FMM results and SPP model predictions, respectively. The green and black vertical dashed lines show the aperture lengths determined by Equations (14) and (15), respectively. $(\mathbf{a}, \mathbf{c})$ The point source is at the center of the aperture, the aperture width is $D=30 \mathrm{~nm}$ and the aperture depth is $h=50 \mathrm{~nm}$ (a) and $30 \mathrm{~nm}$ (c). $(\mathbf{b}, \mathbf{d})$ The distance from the point source to the left end of the aperture is $d=63 \mathrm{~nm}, D=30 \mathrm{~nm}(\mathbf{b})$ and $10 \mathrm{~nm}(\mathbf{d})$ and $h=50 \mathrm{~nm}$. The value of $\Gamma_{\text {rad }}$ in (d) is multiplied by 2 for a clear display.

To understand the numerical results, we used the SPP model to predict the $\Gamma_{\text {total }}$ and $\Gamma_{\text {rad }}$. As shown in Figure 2, the model predictions (circles) agree well with the full-wave a-FMM results (solid curves), which confirms the validity of the model. On the other hand, 
the prediction of the SPP model has an obvious error near the first resonance peak. This shows that in addition to the SPP, other higher-order modes neglected in the model (the field formed by their superposition is called the residual field [38]) also contribute to the antenna radiation. To directly observe the SPP field and the residual field, we calculated the residual field expressed as $\boldsymbol{\Psi}_{\text {res }}=\boldsymbol{\Psi}_{\text {total }}-\boldsymbol{\Psi}_{\mathrm{SPP}}$, where $\boldsymbol{\Psi}_{\text {total }}$ is the total field and $\boldsymbol{\Psi}_{\mathrm{SPP}}$ is the SPP field. Here, the $\boldsymbol{\Psi}_{\text {total }}$ is obtained with the full-wave a-FMM. The SPP coefficients can be extracted from $\boldsymbol{\Psi}_{\text {total }}$ by using the orthogonality relationship $[50,51]$ between the SPP mode and other higher-order modes in the aperture (the latter constituting the $\Psi_{\text {res }}$ ). The calculated SPP field $\left|\mathbf{E}_{\text {SPP }}\right|$ (left column) and the residual field $\left|\mathbf{E}_{\text {res }}\right|$ (right column) obtained at the first four resonance peaks in Figure $2 \mathrm{~b}$ under the point-source excitation are shown in Figure 3. For a direct comparison, all the SPP fields $\left|\mathbf{E}_{S P P}\right|$ are normalized to have a maximum value of one. At the first resonance peak (Figure $3 a, N=0$ ), the residual field is comparable to the SPP field. At other resonance peaks, the residual field is significantly weaker than the SPP field (Figure $3 \mathrm{~b}-\mathrm{d}, M=0$ and $N=M=1$, respectively). This explains the error of the model at the first resonance peak $(N=0)$ in Figure 2 and the high accuracy of the model at other formants.

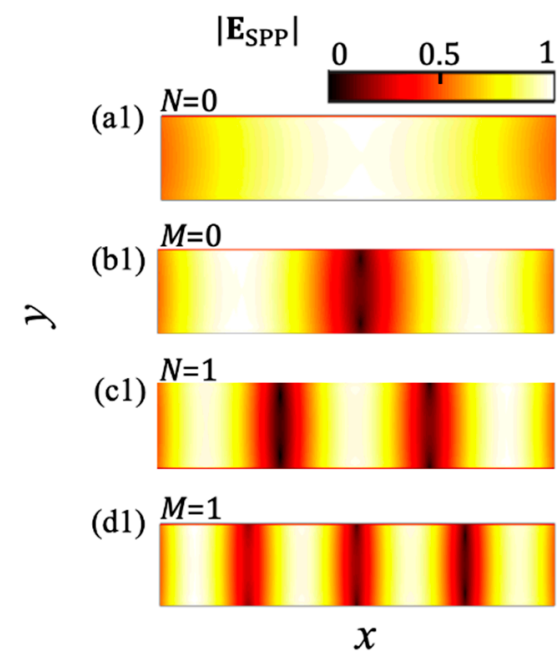

(a2)

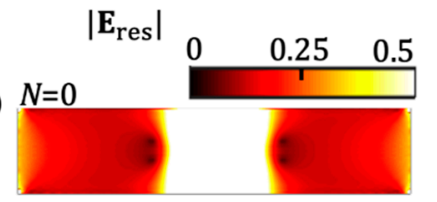

(b2)

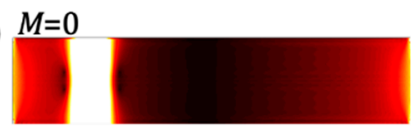

(c2)

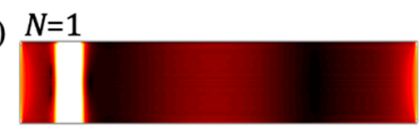

(d2)

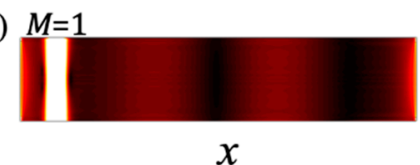

Figure 3. SPP field $\left|\mathbf{E}_{S P P}\right|$ (left column) and the residual field $\left|\mathbf{E}_{\text {res }}\right|$ (right column). (a-d) are obtained at the first four resonance peaks in Figure $2 \mathrm{~b}(L=124,314,514,704 \mathrm{~nm}$, respectively) under the point-source excitation. The electric field amplitude $|\mathbf{E}|=\left(\left|E_{x}\right|^{2}+\left|E_{y}\right|^{2}+\left|E_{z}\right|^{2}\right)^{1 / 2}$ on the $z=h / 2$ plane in the rectangular aperture (width $D=30 \mathrm{~nm}$ ) is shown. For a direct comparison, all the SPP fields $\left|\mathrm{E}_{\mathrm{SPP}}\right|$ are normalized to have a maximum value of 1 .

According to the model Equation (12), when the total emission rate $\Gamma_{\text {total }}$ reaches the maximum, the SPP mode coefficients $b_{1}$ and $b_{2}$ are required to reach the maximum. Equations (8) and (10) show that $b_{1}$ and $b_{2}$ will reach the maximum under one of the following two phase-matching conditions:

$$
\begin{gathered}
k_{0} \operatorname{Re}\left(n_{\text {eff }}\right) L+\arg (r)=2 N \pi, \\
k_{0} \operatorname{Re}\left(n_{\text {eff }}\right) L+\arg (r)=(2 M+1) \pi,
\end{gathered}
$$

where $\operatorname{Re}()$ denotes the real part, $\arg ()$ denotes the argument, and $N$ and $M$ are integers corresponding to different orders of resonance. Equations (14) and (15) are obtained by minimizing $1-u r$ and $1+u r$, respectively, and with the following considerations. Firstly, due to the strong reflection of the SPP at the end of the aperture, $|r|$ is close to one. Secondly, there is $|u|=\exp \left[-k_{0} \operatorname{Im}\left(n_{\text {eff }}\right) L\right] \approx 1$ due to $\operatorname{Im}\left(n_{\text {eff }}\right) \approx 0$ for the SPP that is a propagative mode. For example, for the aperture sizes $(D, h)=(30,50),(30,30),(10,50) \mathrm{nm}$ and at wavelength $\lambda=1 \mu \mathrm{m}$ in the calculations of Figure 2, there are $r=0.859 \exp (-2.029 i), 0.822 \exp (-1.868 i)$ and $0.944 \exp (-2.149 i)$, and $n_{\text {eff }}=2.574+0.059 i, 2.788+0.079 i$ and $4.060+0.133 i$, respec- 
tively. Equations (14) and (15) can be used to determine the length of the aperture at resonance, as shown by the vertical dashed lines in Figure 2. Equations (14) and (15) show that when $L$ is taken so that the emission rate is at the resonance peak, the phase shift experienced by the SPP propagating back and forth in the aperture for one cycle is an integral multiple of $2 \pi$, which makes the SPPs after multiple reflections interfere constructively, i.e., the Fabry-Perot resonance of SPP is formed.

Figure 4 shows the distributions of the dominant electric-field component $E_{y}$ in the aperture excited by the point source at the resonance peak positions in Figure $2 \mathrm{a}, \mathrm{b}$, which are obtained with the full-wave a-FMM [47,48]. It is seen that for the even-order resonances $(N=0,1,2)$, the field $E_{y}$ exhibits a symmetric distribution about the center of the aperture $(x=0)$, while $E_{y}$ exhibits an antisymmetric distribution for the odd-order resonances $(M=0,1,2)$. As shown in Figure $4 \mathrm{a}-\mathrm{c}$, when the point source is located at the center of the aperture $(d=L / 2)$, only the even-order resonant modes are excited. As shown in Figure $4 \mathrm{~d}-\mathrm{i}$, when the point source deviates from the center of the aperture $(d=63 \mathrm{~nm})$, both the even-order and the odd-order resonant modes will be excited, and the field distributions of the excited even-order resonant modes are very close to those when the point source is located at the center of the aperture. As indicated by the phase-matching conditions (14) and (15), the location of these resonant modes should be independent of the location of the emitter. Figure 4 also shows that an interference of two counterpropagating SPPs in the aperture forms the standing wave. With the increase in resonance orders (at the same time, the antenna length $L$ increases), the number of standing-wave nodes in the aperture increases and the intensity of the field decreases. The latter is consistent with the gradual decrease in the spontaneous emission rate $\Gamma_{\text {total }}$ as predicted by the model (see Figure 2).

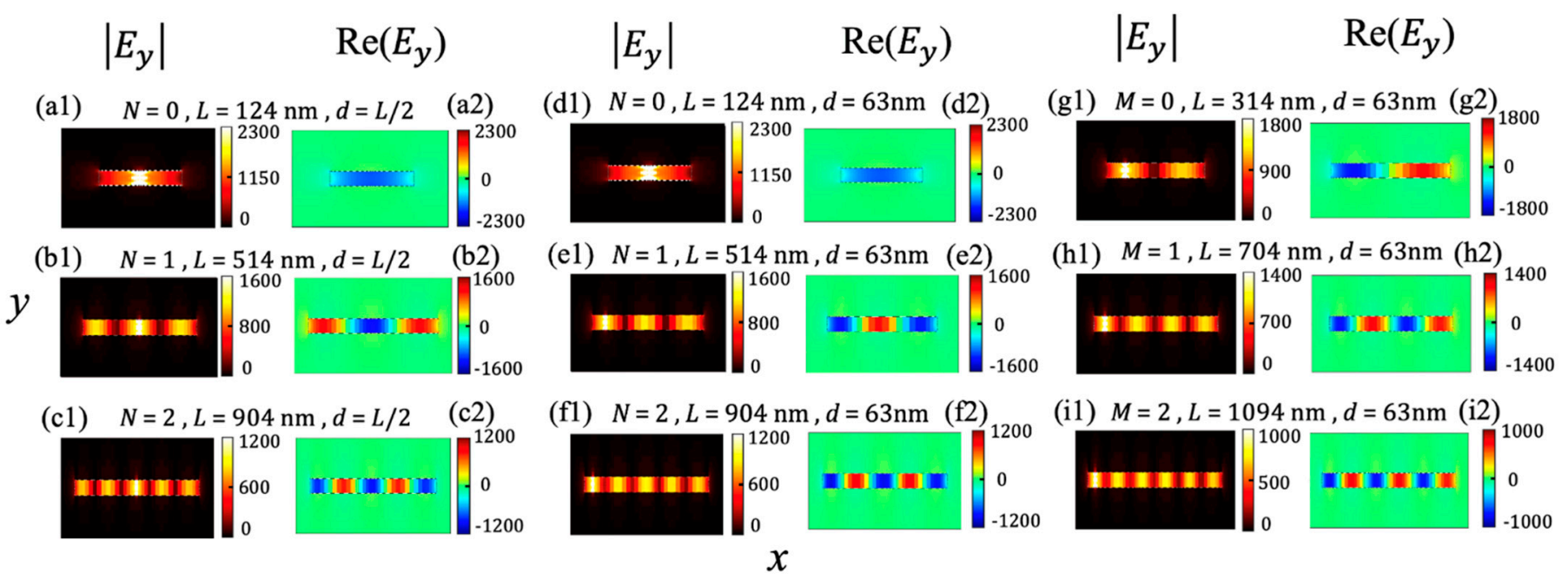

Figure 4. Under the point-source excitation, the distributions of the dominant electric-field component $E_{y}$ at plane $z=h / 2$ in the aperture, which are obtained with the full-wave a-FMM. $(\mathbf{a}-\mathbf{c})$ correspond to the first three resonance peaks $(N=0,1$, $2)$ in Figure 2a. (d-i) correspond to the first six resonance peaks $(N=0,1,2, M=0,1,2)$ in Figure $2 b$. The superimposed dotted lines show the aperture boundary. $E_{y}$ in the figure is normalized (divided by $\Gamma_{\text {water }}$ ).

In addition, compared with the full-wave numerical methods, such as the a-FMM [47,48], the SPP model can reduce the number of numerical calculations. For example, when changing the position of the point source in the aperture, the SPP excitation coefficient $\beta$ by the point source can be calculated with the reciprocity theorem (Equation (5)) without having to solve the Maxwell's equations repeatedly. When changing the aperture length $L$, the SPP model can be executed as well without repeatedly solving the Maxwell's equations. In contrast, if the full-wave a-FMM is used, the Maxwell's equations need to be solved repeatedly when changing the position of the point source and the length of the aperture. Figure 5 shows the results of the normalized total spontaneous emission rate $\Gamma_{\text {total }} / \Gamma_{\text {water }}$ 
obtained with the SPP model depending on the point-source position $d$ (distance from the point source to the left end of the aperture) and the aperture length $L$. It can be seen that if $L$ takes the value determined by the phase-matching conditions of Equations (14) and (15) (vertical dashed lines), with the increase in $L, \Gamma_{\text {total }}$ can have $1,2, \ldots, 6$ maxima in turn (when scanning $d$ ). These maxima correspond to the $1,2, \ldots, 6$ maxima (with the increase in $L$ ) of the dominant electric-field component $\left|E_{y}\right|$ (in the polarization $y$-direction of the point source) of the resonant modes excited in the aperture, and the positions where $\left|E_{y}\right|$ takes the maxima are consistent with the values of $d$ enabling the maxima of $\Gamma_{\text {total }}$ (for instance, $d=63 \mathrm{~nm}$ or $L / 2$ as shown by the white dashed or solid lines in Figure 5 ), as can be seen through a comparison between Figures 4 and 5 . The above-described correspondence between the maxima of $\left|E_{y}\right|$ and the maxima of $\Gamma_{\text {total }}$ is consistent with the prediction of the QNM expansion theory [32,52], in which the resonant modes excited in the aperture can be rigorously defined as the QNMs.

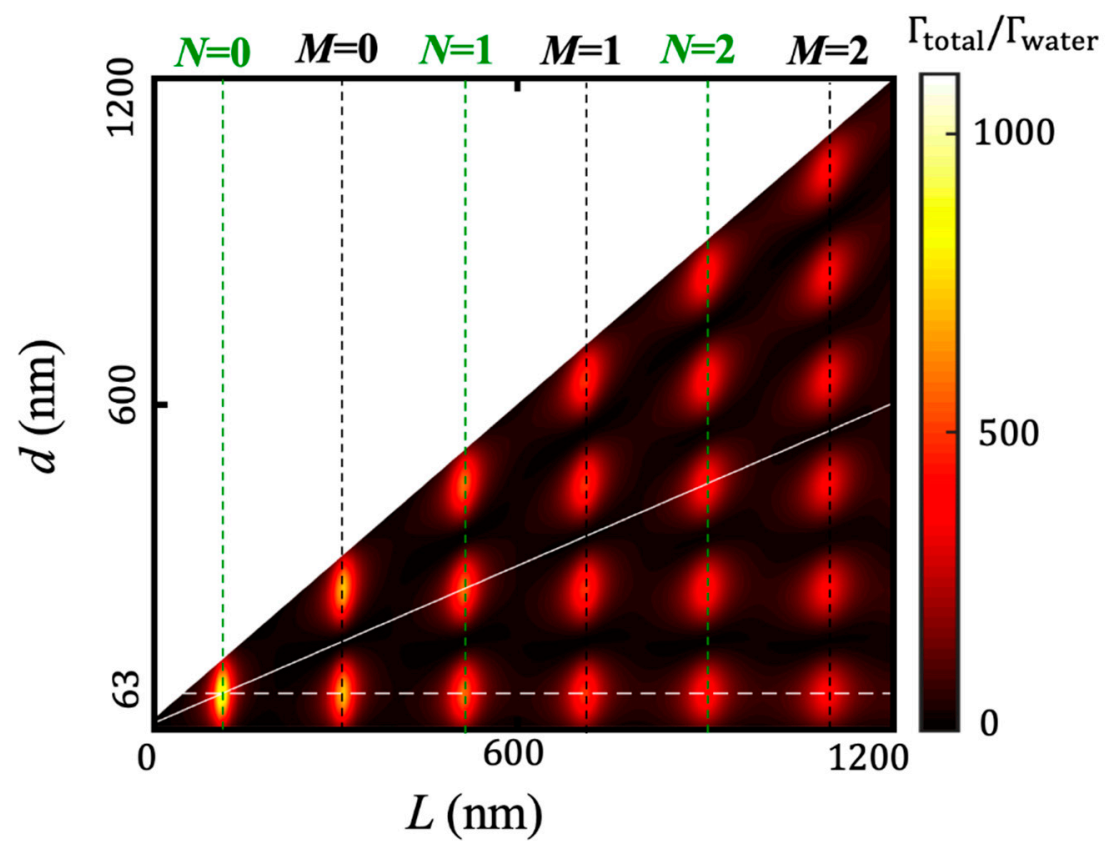

Figure 5. Normalized total spontaneous emission rate $\Gamma_{\text {total }} / \Gamma_{\text {water }}$ depending on the point-source position $d$ (distance from the point source to the left end of the aperture) and the aperture length $L$. The white solid and dashed lines are for $d=L / 2$ (the point source being at the center of the aperture) and $d=63 \mathrm{~nm}$, respectively, corresponding to Figure 2a,b. The green and black vertical dashed lines show the aperture lengths determined by the phase-matching conditions of Equations (14) and (15), respectively. The results are obtained using the SPP model for an aperture width $D=30 \mathrm{~nm}$ and depth $h=50 \mathrm{~nm}$ at $\lambda=1 \mu \mathrm{m}$.

Figure 6 shows the angular distributions of the far-field emission $P(\theta, \phi)=|\mathbf{S}(r, \theta, \phi)| / S_{\text {water }}$ for the rectangular-aperture nanoantenna at the first six resonance peaks of $\Gamma_{\text {rad }}$ (as shown in Figure $2 \mathbf{b})$. $|\mathbf{S}(r, \theta, \phi)|$ is obtained by calculating the modulus $|\mathbf{S}|$ of the time-averaged Poynting vector on a hemisphere in the $z>0$ region (the water side), which is centered at the center of the aperture on the $\mathrm{SiO}_{2}$ substrate surface and has a radius of $r>>\lambda$ (the results on the $z<0$ hemisphere are similar to those on the $z>0$ hemisphere). $\theta$ and $\phi$ are the polar angle and azimuth angle, respectively. $S_{\text {water }}=\Gamma_{\text {water }} /\left(4 \pi r^{2}\right)$ is the average energy-flux density on a sphere of radius $r$ for the point source located in a uniform water environment. Note that the $P(\theta, \phi)$ is asymptotically independent of $r$ as $r$ approaches infinity. $\mathbf{S}(r, \theta, \phi)$ is calculated using the near-to-far field transformation method [53]. This method needs to use the electromagnetic field on a closed surface encompassing the antenna and the point source. The latter can be calculated by using the full-wave a-FMM $[47,48]$ or the SPP model Equation (13). Figure 6 shows that the SPP model (the bottom row) can quantitatively 

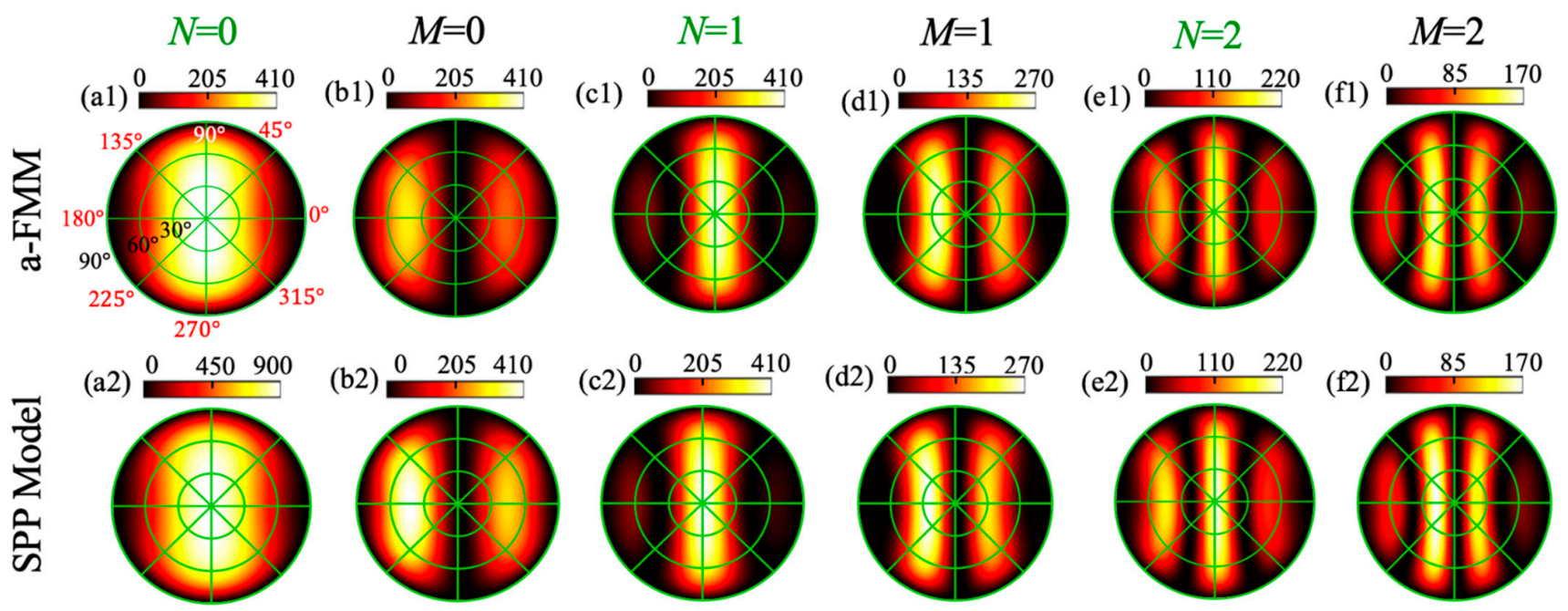

Figure 6. Angular distributions of the far-field emission $P(\theta, \phi)$ of the rectangular-aperture nanoantenna obtained at the antenna resonance (the aperture length $L$ satisfies Equations (14) and (15)) on a hemisphere in $z>0$ region (the water side). (a) $N=0, L=124 \mathrm{~nm}$. (b) $M=0, L=314 \mathrm{~nm}$. (c) $N=1, L=514 \mathrm{~nm}$. (d) $M=1, L=704 \mathrm{~nm}$. (e) $N=2, L=904 \mathrm{~nm}$. (f) $M=2$, $L=1094 \mathrm{~nm}$. The results are obtained at the first six resonance peaks shown in Figure $2 \mathrm{~b}$ for $\lambda=1 \mu \mathrm{m}, D=30 \mathrm{~nm}$ and $d=63 \mathrm{~nm}$. The first and second rows are obtained using the full-wave a-FMM and the SPP model, respectively. The polar angle $\theta$ and azimuthal angle $\phi$ correspond to the superimposed green circles and radial lines, respectively.

\section{Conclusions}

The spontaneous-emission enhancement effect of a single metallic rectangular-aperture optical nanoantenna on a $\mathrm{SiO}_{2}$ substrate is investigated comprehensively and quantitatively. The full-wave a-FMM numerical calculation results show that the rectangular-aperture antenna can achieve a total emission rate enhancement factor $\gamma_{\text {total }}$ up to 5152 and a radiative emission rate enhancement factor $\gamma_{\text {rad }}$ up to 1971 (with an aperture width $D=10 \mathrm{~nm}$, a length $L=84 \mathrm{~nm}$ and a depth $h=50 \mathrm{~nm}$ ), and can achieve a radiation efficiency $\Gamma_{\text {rad }} / \Gamma_{\text {total }}$ up to $69.89 \%$ (for which $\gamma_{\text {total }}=658.81, \gamma_{\text {rad }}=460.43, D=30 \mathrm{~nm}, L=124 \mathrm{~nm}$ and $h=50 \mathrm{~nm}$ ). Moreover, the antenna can also achieve a strong far-field emission within a central angular zone (polar angle $\theta \leq 60^{\circ}$ for instance) that corresponds to a certain numerical aperture of the objective. These properties are of great significance for improving the fluorescence intensity and achieving single-molecule fluorescence detection under high concentrations in physiological environment with the aperture-type nanoantenna.

To explain the numerical results and clarify the underlying physics of the spontaneous emission enhancement of the rectangular-aperture nanoantenna, an intuitive semianalytical SPP model was built based on an intuitive excitation and multiple-scattering process of the SPPs. The model can comprehensively predict the total spontaneous emission rate, the radiative emission rate and the angular distribution of the far-field emission 
of the antenna. A comparison between the prediction of the SPP model and the numerical calculation results of the full-wave a-FMM verifies the validity of the model and shows that for the lowest-order resonance (with a small value of antenna length $L$ ), the residual field other than the SPP considered in the model also contributes to antenna radiation. Two phase-matching conditions are derived from the model to predict the antenna resonance and the resultant enhancement of the spontaneous emission rate and show that the enhancement of the spontaneous emission rate comes from a Fabry-Perot resonance of the SPP. In addition, when scanning the position of the point source and the length of the aperture antenna, the SPP model shows a superior computational efficiency compared to the full-wave numerical method. The Fabry-Perot SPP model presented in this paper may be extended to other types of optical nanoantennas that support resonance formed by a multiple scattering of propagative waveguide modes $[21,22,59,60]$. For instance, FabryPerot models can be established by considering the multiple scattering of the fundamental SPP mode for a single-nanowire antenna [61], dipole nanoantenna [38] and nanoparticle with an arbitrary shape [62], or fundamental photonic mode for a semiconductor-nanowire antenna [63]. The contribution of the residual field [64] may be further considered in the model to improve its accuracy. The SPP model may have extended applications in various fields such as biosensing $[6,56,65]$, light-matter interaction enhancement $[21,42,66]$, etc.

Author Contributions: Conceptualization, H.L.; methodology, H.L.; software, H.L.; validation, X.Z. (Xinyue Zhang); formal analysis, X.Z. (Xinyue Zhang); investigation, X.Z. (Xinyue Zhang), X.Z. (Xuelin Zhai), C.T., N.W., Y.Z. and H.L.; resources, H.L.; data curation, X.Z. (Xinyue Zhang); writingoriginal draft preparation, X.Z. (Xinyue Zhang); writing-review and editing, X.Z. (Xinyue Zhang) and H.L.; visualization, X.Z. (Xinyue Zhang); supervision, H.L.; project administration, H.L.; funding acquisition, H.L. All authors have read and agreed to the published version of the manuscript.

Funding: National Natural Science Foundation of China $(62075104,61775105)$.

Data Availability Statement: The data presented in this paper are available from the authors upon reasonable request.

Conflicts of Interest: The authors declare no conflict of interest.

\section{References}

1. Ming, T.; Chen, H.; Jiang, R.; Li, Q.; Wang, J. Plasmon-controlled fluorescence: Beyond the intensity enhancement. J. Phys. Chem. Lett. 2012, 3, 191-202. [CrossRef]

2. Agio, M. Optical antennas as nanoscale resonators. Nanoscale 2012, 4, 692-706. [CrossRef]

3. Acuna, G.; Möller, F.; Holzmeister, P.; Beater, S.; Lalkens, B.; Tinnefeld, P. Fluorescence enhancement at docking sites of DNA-directed self-assembled nanoantennas. Science 2012, 338, 506-510. [CrossRef] [PubMed]

4. Sergei, K.; Ulf, H.; Rogobete, L.; Sandoghdar, V. Enhancement of single-molecule fluorescence using a gold nanoparticle as an optical nanoantenna. Phys. Rev. Lett. 2006, 97, 017402.

5. Punj, D.; Ghenuche, P.; Moparthi, S.B.; de Torres, J.; Grigoriev, V.; Rigneault, H.; Wenger, J. Plasmonic antennas and zero-mode waveguides to enhance single molecule fluorescence detection and fluorescence correlation spectroscopy toward physiological concentrations. Nat. Nanotechnol. 2014, 6, 268-282. [CrossRef]

6. Zhao, C.; Liu, Y.; Yang, J.; Zhang, J. Single-molecule detection and radiation control in solutions at high concentrations via a heterogeneous optical slot antenna. Nanoscale 2014, 6, 9103-9109. [CrossRef]

7. Genet, C.; Ebbesen, T.W. Light in tiny holes. Nature 2007, 445, 39-46. [CrossRef] [PubMed]

8. Levene, M.J.; Korlach, J.; Turner, S.W.; Foquet, M.; Craighead, H.G.; Webb, W.W. Zero-mode waveguides for single-molecule analysis at high concentrations. Science 2003, 299, 682-686. [CrossRef]

9. Alam, M.S.; Karim, F.; Zhao, C. Single-molecule detection at high concentrations with optical aperture nanoantennas. Nanoscale 2016, 8, 9480-9487. [CrossRef]

10. Fujimoto, K.; Morita, Y.; Iino, R.; Tomishige, M.; Shintaku, H.; Kotera, H.; Yokokawa, R. Simultaneous observation of kinesindriven microtubule motility and binding of adenosine triphosphate using linear zero-mode waveguides. ACS Nano 2018, 12, 11975-11985. [CrossRef]

11. Moran-Mirabal, J.M.; Craighead, H.G. Zero-mode waveguides: Sub-wavelength nanostructures for single molecule studies at high concentrations. Methods 2008, 46, 11-17. [CrossRef] [PubMed]

12. Barnes, W.L.; Dereux, A.; Ebbesen, T.W. Surface plasmon subwavelength optics. Nature 2003, 424, 824-830. [CrossRef] [PubMed]

13. Gramotnev, D.K.; Bozhevolnyi, S.I. Plasmonics beyond the diffraction limit. Nat. Photonics 2010, 4, 83-91. [CrossRef]

14. Pelton, M. Modified spontaneous emission in nanophotonic structures. Nat. Photonics 2015, 9, 427-435. [CrossRef] 
15. Miyake, T.; Tanii, T.; Sonobe, H.; Akahori, R.; Shimamoto, N.; Ueno, T.; Funatsu, T.; Ohdomari, I. Real-time imaging of singlemolecule fluorescence with a zero-mode waveguide for the analysis of protein-protein interaction. Anal. Chem. 2008, 80, 6018-6022. [CrossRef]

16. Wenger, J.; Lenne, P.-F.; Popov, E.; Rigneault, H.; Dintinger, J.; Ebbesen, T.W. Single molecule fluorescence in rectangular nano-apertures. Opt. Express 2005, 13, 7035-7044. [CrossRef]

17. Zambrana-Puyalto, X.; Ponzellini, P.; Maccaferri, N.; Tessarolo, E.; Pelizzo, M.G.; Zhang, W.; Barbillon, G.; Lu, G.; Garoli, D. A hybrid metal-dielectric zero mode waveguide for enhanced single molecule detection. Chem. Commun. 2019, 55, 9725-9728. [CrossRef]

18. Aouani, H.; Mahboub, O.; Devaux, E.; Rigneault, H.; Ebbesen, T.W.; Wenger, J. Large molecular fluorescence enhancement by a nanoaperture with plasmonic corrugations. Opt. Express 2011, 19, 13056-13062. [CrossRef] [PubMed]

19. Park, Q.-H. Optical antennas and plasmonics. Contemp. Phys. 2009, 50, 407-423. [CrossRef]

20. Seo, M.; Adam, A.; Kang, J.; Lee, J.; Ahn, K.; Park, Q.H.; Planken, P.; Kim, D. Near field imaging of terahertz focusing onto rectangular apertures. Opt. Express 2008, 16, 20484-20489. [CrossRef]

21. El Eter, A.; Hameed, N.M.; Baida, F.I.; Salut, R.; Filiatre, C.; Nedeljkovic, D.; Atie, E.; Bole, S.; Grosjean, T. Fiber-integrated optical nano-tweezer based on a bowtie-aperture nano-antenna at the apex of a SNOM tip. Opt. Express 2014, 22, 10072-10080. [CrossRef] [PubMed]

22. Jin, E.X.; Xu, X. Obtaining super resolution light spot using surface plasmon assisted sharp ridge nanoaperture. Appl. Phys. Lett. 2005, 86, 111106. [CrossRef]

23. Zhang, J.; Zhang, W.; Zhu, X.; Yang, J.; Xu, J.; Yu, D. Resonant slot nanoantennas for surface plasmon radiation in optical frequency range. Appl. Phys. Lett. 2012, 100, 241115. [CrossRef]

24. Yousif, B.B.; Samra, A.S. Modeling of optical nanoantennas. Phys. Res. Int. 2012, 2012, 321075. [CrossRef]

25. Ponzellini, P.; Zambrana-Puyalto, X.; Maccaferri, N.; Lanzanò, L.; De Angelis, F.; Garoli, D. Plasmonic zero mode waveguide for highly confined and enhanced fluorescence emission. Nanoscale 2018, 10, 17362-17369. [CrossRef]

26. Chen, K.; Razinskas, G.; Vieker, H.; Gross, H.; Wu, X.; Beyer, A.; Gölzhäuser, A.; Hecht, B. High-Q, low-mode-volume and multiresonant plasmonic nanoslit cavities fabricated by helium ion milling. Nanoscale 2018, 10, 17148-17155. [CrossRef] [PubMed]

27. Kotnala, A.; Ding, H.; Zheng, Y. Enhancing single-molecule fluorescence spectroscopy with simple and robust hybrid nanoapertures. ACS Photonics 2021, 8, 1673-1682. [CrossRef]

28. Mahdavi, F.; Blair, S. Nanoaperture fluorescence enhancement in the ultraviolet. Plasmonics 2010, 5, 169-174. [CrossRef]

29. Flauraud, V.; Regmi, R.; Winkler, P.M.; Alexander, D.T.; Rigneault, H.; Van Hulst, N.F.; García-Parajo, M.F.; Wenger, J.; Brugger, J. In-plane plasmonic antenna arrays with surface nanogaps for giant fluorescence enhancement. Nano Lett. 2017, 17, 1703-1710. [CrossRef]

30. Ghenuche, P.; Mivelle, M.; de Torres, J.; Moparthi, S.B.; Rigneault, H.; Van Hulst, N.F.; García-Parajó, M.F.; Wenger, J. Matching nanoantenna field confinement to FRET distances enhances Forster energy transfer rates. Nano Lett. 2015, 15, 6193-6201. [CrossRef] [PubMed]

31. Punj, D.; Mivelle, M.; Moparthi, S.B.; Van Zanten, T.S.; Rigneault, H.; Van Hulst, N.F.; Garcia-Parajo, M.F.; Wenger, J. A plasmonic 'antenna-in-box' platform for enhanced single-molecule analysis at micromolar concentrations. Nat. Nanotechnol. 2013, 8, 512-516. [CrossRef] [PubMed]

32. Lalanne, P.; Yan, W.; Vynck, K.; Sauvan, C.; Hugonin, J.P. Light interaction with photonic and plasmonic resonances. Laser Photonics Rev. 2018, 12, 1700113. [CrossRef]

33. Ching, E.; Leung, P.; van den Brink, A.M.; Suen, W.; Tong, S.; Young, K. Quasinormal-mode expansion for waves in open systems. Rev. Mod. Phys. 1998, 70, 1545-1554. [CrossRef]

34. Teperik, T.; Popov, V.; García de Abajo, F.J. Giant light absorption by plasmons in a nanoporous metal film. Phys. Status Solidi A 2005, 202, 362-366. [CrossRef]

35. Cetin, A.E.; Turkmen, M.; Aksu, S.; Etezadi, D.; Altug, H. Multi-resonant compact nanoaperture with accessible large nearfields. Appl. Phys. B Lasers Opt. 2015, 118, 29-38. [CrossRef]

36. Wan, Y.; An, Y.; Tao, Z.; Deng, L. Manipulation of surface plasmon resonance of a graphene-based Au aperture antenna in visible and near-infrared regions. Opt. Commun. 2018, 410, 733-739. [CrossRef]

37. Palik, E.D. Handbook of Optical Constants of Solids; Academic: San Diego, CA, USA, 1985.

38. Jia, H.; Liu, H.; Zhong, Y. Role of surface plasmon polaritons and other waves in the radiation of resonant optical dipole antennas. Sci. Rep. 2015, 5, 8456. [CrossRef]

39. Purcell, E.M. Spontaneous emission probabilities at radio frequencies. Phys. Rev. 1946, 69, 681.

40. Bogdanov, S.I.; Shalaginov, M.Y.; Lagutchev, A.S.; Chiang, C.-C.; Shah, D.; Baburin, A.S.; Ryzhikov, I.A.; Rodionov, I.A.; Kildishev, A.V.; Boltasseva, A. Ultrabright room-temperature sub-nanosecond emission from single nitrogen-vacancy centers coupled to nanopatch antennas. Nano Lett. 2018, 18, 4837-4844. [CrossRef] [PubMed]

41. Lodahl, P.; Mahmoodian, S.; Stobbe, S. Interfacing single photons and single quantum dots with photonic nanostructures. Rev. Mod. Phys. 2015, 87, 347-400. [CrossRef]

42. Giannini, V.; Fernández-Domínguez, A.I.; Heck, S.C.; Maier, S.A. Plasmonic nanoantennas: Fundamentals and their use in controlling the radiative properties of nanoemitters. Chem. Rev. 2011, 111, 3888-3912. [CrossRef] 
43. Muskens, O.; Giannini, V.; Sánchez-Gil, J.A.; Gómez Rivas, J. Strong enhancement of the radiative decay rate of emitters by single plasmonic nanoantennas. Nano Lett. 2007, 7, 2871-2875. [CrossRef]

44. Kinkhabwala, A.; Yu, Z.; Fan, S.; Avlasevich, Y.; Müllen, K.; Moerner, W.E. Large single-molecule fluorescence enhancements produced by a bowtie nanoantenna. Nat. Photonics 2009, 3, 654-657. [CrossRef]

45. Ma, R.M.; Oulton, R.F.; Sorger, V.J.; Zhang, X. Plasmon lasers: Coherent light source at molecular scales. Laser Photonics Rev. 2013, 7,1-21. [CrossRef]

46. Tsakmakidis, K.L.; Boyd, R.W.; Yablonovitch, E.; Zhang, X. Large spontaneous-emission enhancements in metallic nanostructures: Towards LEDs faster than lasers. Opt. Express 2016, 24, 17916-17927. [CrossRef] [PubMed]

47. Hugonin, J.P.; Lalanne, P. Perfectly matched layers as nonlinear coordinate transforms: A generalized formalization. J. Opt. Soc. Am. A 2005, 22, 1844-1849. [CrossRef]

48. Liu, H. DIF CODE for Modeling Light Diffraction in Nanostructures; Nankai University: Tianjin, China, 2010.

49. Zhu, J.; Zhu, T.; Jia, H.; Zhong, Y.; Liu, H. Intuitive analysis of subwavelength plasmonic trench waveguide. J. Lightwave Technol. 2019, 37, 1345-1351. [CrossRef]

50. Vassallo, C. Optical Waveguide Concepts; Elsevier: Amsterdam, The Netherlands, 1991.

51. Jia, H.; Lalanne, P.; Liu, H. Comprehensive surface-wave description for the nano-scale energy concentration with resonant dipole antennas. Plasmonics 2016, 11, 1025-1033. [CrossRef]

52. Sauvan, C.; Hugonin, J.-P.; Maksymov, I.S.; Lalanne, P. Theory of the spontaneous optical emission of nanosize photonic and plasmon resonators. Phys. Rev. Lett. 2013, 110, 237401. [CrossRef] [PubMed]

53. Yang, J.; Hugonin, J.-P.; Lalanne, P. Near-to-far field transformations for radiative and guided waves. ACS Photonics 2016, 3 , 395-402. [CrossRef]

54. Taminiau, T.H.; Stefani, F.D.; van Hulst, N.F. Optical nanorod antennas modeled as cavities for dipolar emitters: Evolution of sub-and super-radiant modes. Nano Lett. 2011, 11, 1020-1024. [CrossRef] [PubMed]

55. Zhai, X.; Wang, N.; Zhong, Y.; Liu, H. Broadband enhancement of the spontaneous emission by a split-ring nanoantenna: Impact of azimuthally propagating surface plasmon polaritons. IEEE J. Sel. Top. Quantum Electron. 2021, 27, 4600815. [CrossRef]

56. Bauch, M.; Toma, K.; Toma, M.; Zhang, Q.; Dostalek, J. Plasmon-enhanced fluorescence biosensors: A review. Plasmonics 2014, 9 , 781-799. [CrossRef]

57. Aouani, H.; Mahboub, O.; Bonod, N.; Devaux, E.; Popov, E.; Rigneault, H.; Ebbesen, T.W.; Wenger, J. Bright unidirectional fluorescence emission of molecules in a nanoaperture with plasmonic corrugations. Nano Lett. 2011, 11, 637-644. [CrossRef]

58. Aouani, H.; Mahboub, O.; Devaux, E.; Rigneault, H.; Ebbesen, T.W.; Wenger, J. Plasmonic antennas for directional sorting of fluorescence emission. Nano Lett. 2011, 11, 2400-2406. [CrossRef]

59. Chang, Y.-T.; Lai, Y.-C.; Li, C.-T.; Chen, C.-K.; Yen, T.-J. A multi-functional plasmonic biosensor. Opt. Express 2010, 18, 9561-9569. [CrossRef]

60. Tobing, L.Y.; Goh, G.-Y.; Mueller, A.D.; Ke, L.; Luo, Y.; Zhang, D.-H. Polarization invariant plasmonic nanostructures for sensing applications. Sci. Rep. 2017, 7, 7539. [CrossRef]

61. Liu, C.; Liu, H.; Zhong, Y. Impact of surface plasmon polaritons and other waves on the radiation of a dipole emitter close to a metallic nanowire antenna. Opt. Express 2014, 22, 25539-25549. [CrossRef] [PubMed]

62. Hasan, S.B.; Filter, R.; Ahmed, A.; Vogelgesang, R.; Gordon, R.; Rockstuhl, C.; Lederer, F. Relating localized nanoparticle resonances to an associated antenna problem. Phys. Rev. B 2011, 84, 195405. [CrossRef]

63. Friedler, I.; Sauvan, C.; Hugonin, J.P.; Lalanne, P.; Claudon, J.; Gérard, J.M. Solid-state single photon sources: The nanowire antenna. Opt. Express 2009, 17, 2095-2110. [CrossRef]

64. Liu, H.; Lalanne, P. Light scattering by metallic surfaces with subwavelength patterns. Phys. Rev. B 2010, 82, 115418. [CrossRef]

65. Zhang, L.; Fu, Q.; Tan, Y.; Li, X.; Deng, Y.; Zhou, Z.-K.; Zhou, B.; Xia, H.; Chen, H.; Qiu, C.-W. Metaoptronic multiplexed interface for probing bioentity behaviors. Nano Lett. 2021, 21, 2681-2689. [CrossRef] [PubMed]

66. Zhang, W.; You, J.-B.; Liu, J.; Xiong, X.; Li, Z.; Png, C.E.; Wu, L.; Qiu, C.-W.; Zhou, Z.-K. Steering room-temperature plexcitonic strong coupling: A diexcitonic perspective. Nano Lett. 2021, 21, 8979-8986. [CrossRef] [PubMed] 